${ }^{1}$ Institute for Atmospheric Physics, Johannes Gutenberg University Mainz, Mainz, Germany

${ }^{2}$ Institut für Physik der Atmosphäre, Deutsches Zentrum für Luft- und Raumfahrt (DLR), Oberpfaffenhofen, Germany

${ }^{3}$ Department of Atmospheric Chemistry, Max Planck Institute for Chemistry, Mainz, Germany

${ }^{4}$ Institute for Meteorology and Climate Research, Karlsruhe Institute of Technology (KIT), Karlsruhe, Germany

${ }^{5}$ Institute of Physics and Meteorology, University of Hohenheim, Hohenheim, Germany

${ }^{6}$ MeteoSwiss, Zürich, Switzerland

\title{
Multi-model simulations of a convective situation in low-mountain terrain in central Europe
}

\author{
J. Trentmann ${ }^{1}$, C. Keil ${ }^{2}$, M. Salzmann ${ }^{3}$, C. Barthlott ${ }^{4}$, H.-S. Bauer ${ }^{5}$, T. Schwitalla ${ }^{5}$, \\ M. G. Lawrence ${ }^{3}$, D. Leuenberger ${ }^{6}$, V. Wulfmeyer ${ }^{5}$, U. Corsmeier ${ }^{4}$, C. Kottmeier ${ }^{4}$, H. Wernli ${ }^{1}$ \\ With 4 Figures
}

Received 31 October 2007; Accepted 19 May 2008

Published online 20 August 2008 (C) Springer-Verlag 2008

\begin{abstract}
Summary
The goal of the present study is to investigate the variability of simulated convective precipitation by three convectionresolving models using different set-ups and initial and boundary conditions. The COSMO, MM5 and WRF models have been used to simulate the atmospheric situation on 12 July 2006, when local convection occurred in central Europe under weak synoptic forcing. The focus of this investigation is on the convective precipitation in the northern Black Forest in South-West Germany. The precipitation fields from the nine model simulations differ considerably. Six simulations capture the convective character of the event. However, they differ considerably in the location and timing of the intense convective cells. Only one model simulation captures the early onset of precipitation; in all other simulations, the onset of convective precipitation is delayed by up to five hours. All model simulations significantly underpredict the amount of surface precipitation compared to gauge-adjusted radar observations. The simulated diurnal cycles show maximum CAPE and minimum CIN values in the early afternoon. The different onset times of precipitation in the model simulations are shifted in accordance to the simulated diurnal cycles of CAPE and
\end{abstract}

Correspondence: Jörg Trentmann, Johannes Gutenberg University Mainz, Mainz, Germany (E-mail: jtrent@uni-mainz.de)
CIN. In the simulations with an early onset of precipitation maximum CAPE and minimum CIN values also appear early. The amount of simulated precipitation, however, does not correlate with CAPE or CIN.

\section{Introduction}

In mid-latitude mountainous regions, convective precipitation is the dominant form of summertime precipitation. Forecasting convective precipitation remains a challenge for current state-of-the-art numerical weather prediction (NWP) models. In low-mountain regions, smallscale local flow systems induced by topography determine the timing and location of convection. Only very recently, the spatial resolution of NWP models has been increased to an extent that the processes associated with the initiation and the evolution of deep convection can be explicitly resolved. Studies of the predictability of convective events in convection-resolving models indicate a significant impact of initial and lateral boundary conditions on the simulation 
of convective precipitation for some situations (Hohenegger et al. 2006; Didone et al. 2007). Additional assimilation of water vapor data (e.g., from lidar or GPS) helps to reduce errors in the initial moisture field (Grzeschik et al. 2008) and can have a positive impact on the simulation of convection initiation (Wulfmeyer et al. 2006). Also, the assimilation of radar data (e.g., via latent heat nudging) can improve forecasts by triggering convection in the model at the right time and location (Leuenberger and Rossa 2007).

In the present work we conduct a case study and evaluate the performance of state-of-the-art convection-resolving models under convective conditions in low-mountain terrain in Central Europe. The goal is to improve the understanding of the processes that initiate convection in convection-resolving models and to test whether the convection indices CAPE and CIN are applicable to understanding the initiation of convection in such numerical models.

\section{Participating models}

We compare model results from three different non-hydrostatic numerical models: the operational weather forecast model developed by the Consortium for Small-scale Modeling, the COSMO model (formerly known as the Local Model, LM (Steppeler et al. 2003; Schaettler et al. 2005)), the PSU/NCAR Mesoscale Model (Grell et al. 1995) (known as MM5), and the Weather Research and Forecasting (WRF) model (Skamarock et al. 2005). All model simulations were conducted without parameterization of deep convection, i.e., the initiation and evolution of deep convection in the model simulations is calculated explicitly. Details about the different models and their setups are given in Table 1 and in the following paragraphs.

Results from simulations with the COSMO model are contributed by three groups using different technical setups. The model simulations at the Institute for Atmospheric Physics at the University of Mainz (IPA) were conducted with the COSMO model Version 4.0 using a model domain of $371 \times 351 \times 50$ grid points with a horizontal grid point spacing of $0.025^{\circ}$ (about $2.8 \mathrm{~km}$ ). All models used in the present study employ a vertically staggered grid with $n$ layers and $n+1$ levels. The vertical velocity and turbulent quantities (e.g., TKE) are defined on model layer interfaces (i.e., levels), while all other prognostic variables are defined in the center of the model layers. We only report the number of vertical layers used in the model simulations. Hourly operational COSMO-EU analyses (on a $0.0625^{\circ}$ grid, provided by the German Weather Service (DWD)) served as initial and boundary conditions. The dynamical equations were solved applying a 3rd-order Runge-Kutta scheme using a timestep of $30 \mathrm{sec}$. Shallow convection was parameterized using a modified Tiedke-Scheme and cloud microphysics with a single-moment scheme including graupel. Here, results from two out of several simulations for this case will be presented. The simulation IPA-01 was started at 07 UTC on 12 July 2006, while simulation IPA02 on the same day already at 00 UTC.

The model simulations at the Institute for Meteorology and Climate Research in Karlsruhe (IMK) were conducted using the COSMO model Version 3.19 with a model domain of $121 \times 101$ horizontal grid points (grid point spacing $0.025^{\circ}$ ) and 40 vertical layers. The Leapfrog scheme was used with a timestep of $12 \mathrm{sec}$. Shallow convection was parameterized. The simulation was ini-

Table 1. Model setups used by the participating groups

\begin{tabular}{llllll}
\hline & IPA & IMK & DLR/MS & IPM & MPI-C \\
\hline Model & COSMO, V4.0 & COSMO, V3.19 & COSMO, V3.21 & MM5 & WRF, V2.2 \\
Hor. Resolution & $2.8 \mathrm{~km}$ & $2.8 \mathrm{~km}$ & $2.8 \mathrm{~km}$ & $2.8 / 8.4 \mathrm{~km}$ & $2.0 / 10.0 \mathrm{~km}$ \\
\# hor. grid points & $371 \times 351$ & $121 \times 101$ & $320 \times 350$ & $343 \times 343$ & $171 \times 171$ \\
\# vert. layer & 50 & 40 & 50 & 36 & $54 / 34$ \\
Initial/bd. data & LME analysis & GME analysis/ & COSMO LEPS & ECMWF & NCEP/ECMWF \\
Model start & forecast & forecast & analysis & analysis \\
& & 00 UTC & 06 UTC & 06 UTC & 06 UTC \\
\hline
\end{tabular}


tialized at 00 UTC on 12 July with DWD-GME analysis data. For the lateral boundary conditions, GME forecast data were used.

The model simulations at DLR/MeteoSwiss (DLR/MS) were performed using the COSMO model, Version 3.21, also with a horizontal grid point spacing of $0.025^{\circ}$. For initial and boundary conditions, the results from the COSMO-LEPS ensemble prediction system were used (Molteni et al. 2001). The model simulations were started at 06 UTC on 12 July 2006 using initial and boundary data from COSMO-LEPS forecasts started at 12 UTC the previous day. In total, 10 simulations were conducted using different initial and boundary data. Conventional observations, like radiosoundings and surface observations, were assimilated in all ten simulations from 06 to 14 UTC. For the present work, three representative simulations were selected. A detailed analysis of all simulations is presented in Keil et al. (2007).

Model simulations using the MM5 (Grell et al. 1995) were conducted at the Institute of Physics and Meteorology (IPM) at the University of Hohenheim. They were conducted using two 2way nested grids with grid spacings of $8.4 \mathrm{~km}$ and $2.8 \mathrm{~km}$, respectively. The starting time of the simulations was 06 UTC on 12 July 2006. The innermost domain consists of $343 \times 343$ grid points and 36 vertical layers. Initial and boundary conditions were taken from operational ECMWF analyses (retrieved at $0.25^{\circ} \times 0.25^{\circ}$ horizontal resolution). The chosen physical parameterizations include the Reisner 2 cloud microphysical scheme, the MRF boundary layer scheme, and a 5-layer soil model. No parameterization of shallow convection was employed. Further information on the model and a detailed analysis of model results for convective situations can be found in Schwitalla et al. (2008).

The WRF model, Version 2.2, with the ARW (Advanced Research WRF) dynamical core was used at the Max Planck Institute for Chemistry (MPI-C) in Mainz. A total of seven runs have been performed, two of which are included in this study. In both runs, two sub-domains with $10 \mathrm{~km}$ and $2 \mathrm{~km}$ horizontal resolution and domain sizes of $112 \times 97$ and $171 \times 171$ grid points, respectively, were recursively nested into a coarse domain with $30 \mathrm{~km}$ resolution. The model simulations were initiated at 06 UTC on 12 July
2006. The first of these runs (MPI-NCEP) was driven with NCEP Final Analysis (FNL) Data (retrieved at $1^{\circ} \times 1^{\circ}, 27$ vertical levels) with 54 vertical layers. The second simulation (MPIECMWF) was driven with ECMWF analysis (retrieved at $1.25^{\circ} \times 1.25^{\circ}, 16$ vertical levels) using 34 vertical layers. The Lin microphysics parametrization and the Yonsei State University (YSU) planetary boundary layer parametrization were used in both runs. No parameterization of shallow convection was employed. The two simulations were selected to reflect the variability in terms of the simulated precipitation field in the seven WRF model sensitivity simulations.

\section{Case study: 12 July 2006}

The performance of the different model simulations will be investigated for 12 July 2006 during one of the intensive observation periods of the PRINCE (Prediction, identification, and tracking of convective cells) experiment. This day was characterized by weak large scale forcing over Europe and the formation of single cell convection in the early afternoon over mountainous regions across central Europe (Fig. 1, left). Here, we focus on the situation in south-west Germany.

At around local noon, isolated convective cells that lasted for about three hours formed in the northern Black Forest. The precipitation field derived from gauge-adjusted radar measurements between 09 and 19 UTC shows an area of convective precipitation in the Murg Valley north of Freudenstadt $\left(8.42^{\circ} \mathrm{E}, 48.47^{\circ} \mathrm{N}\right)$ with a maximum precipitation amount of $58 \mathrm{~mm}$ within $10 \mathrm{hrs}$ (Fig. 1, right). Ground-based wind-LIDAR measurements (not shown) obtained at Hornisgrinde $(1177 \mathrm{~m}$ asl, the highest peak in the northern Black Forest) revealed that horizontal wind convergence along the mountain crest, presumably due to thermally-induced mountain wind systems, was responsible for the initiation of these convective cells. Afternoon values of CAPE derived from radiosoundings exceeded $2000 \mathrm{~J} \mathrm{~kg}^{-1}$. Further information on the experimental results of PRINCE can be found in Groenemeijer et al. (2008). Here, we focus on the variability of precipitation in multi-model simulations for this convective event in the northern Black Forest. 

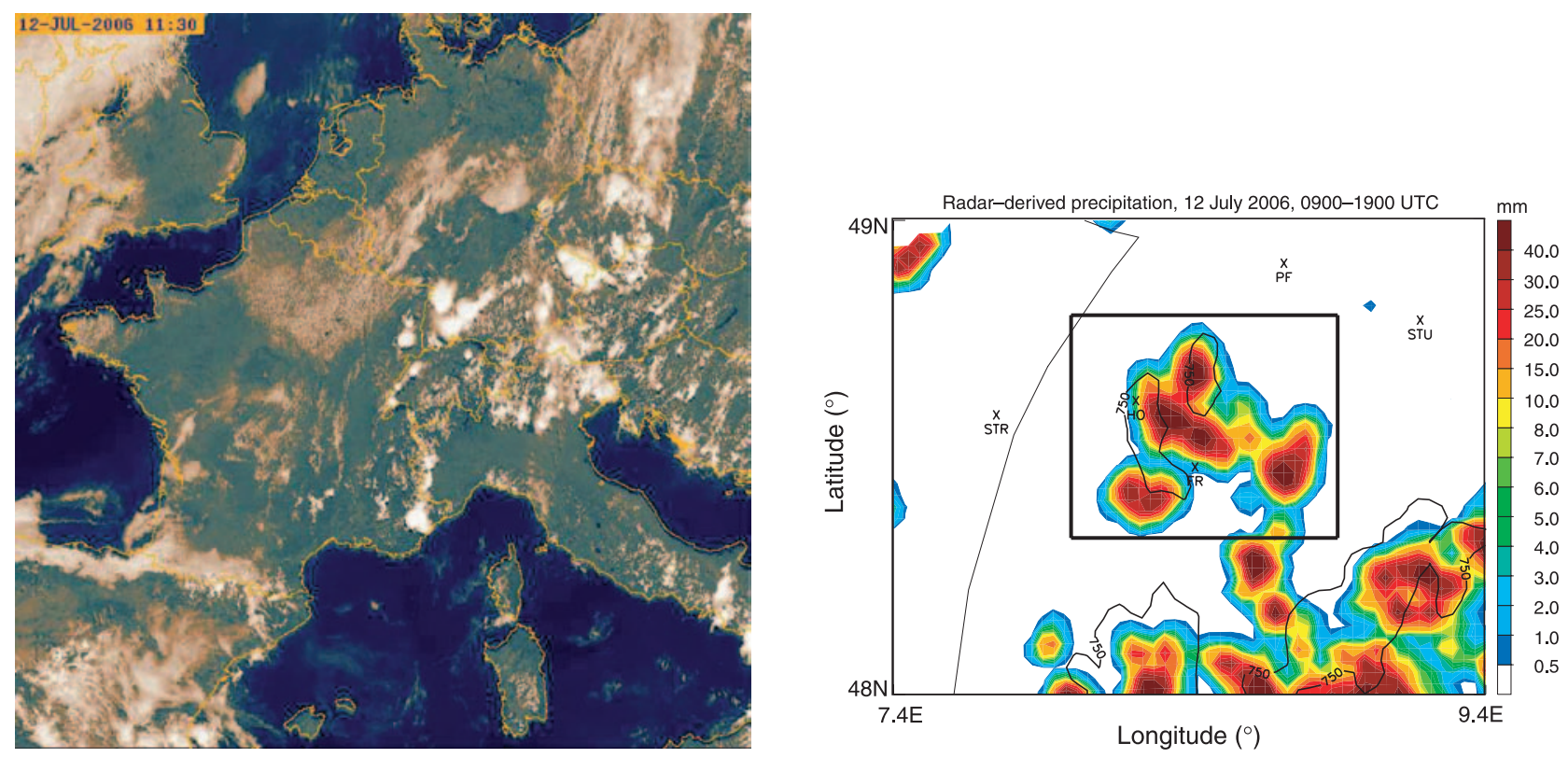

Fig. 1. Left: Meteosat Second Generation (MSG) visible satellite image at 1130 UTC on 12 July 2006. Right: Radar-derived surface precipitation (RW product by DWD) accumulated between 09 UTC and 19 UTC on 12 July 2006. The radar data (originally provided with a horizontal resolution of $1 \mathrm{~km}$ ) were gridded on a $2.8 \mathrm{~km}$ horizontal grid. The thin black contour represents the German-French border, thick black contours represent the $750 \mathrm{~m}$-isosurface of the topography. The black rectangle indicates the region where the spatial averaging was performed for detailed analyses. Also indicated are major cities and landmarks: Strasbourg (STR), Hornisgrinde (HO), Freudenstadt (FR), Pforzheim (PF), and Stuttgart (STU)

\section{Model results}

\subsection{Surface precipitation}

Figure 2 shows the simulated surface precipitation accumulated between 09 and 19 UTC on 12 July 2006 in the northern Black Forest for nine different model simulations. There is a large variability of the simulated precipitation pattern between the three different models. However, there is also a significant spread between the results obtained from the same model using different initial and lateral boundary conditions and technical setups. In principle, all models are able to simulate convective precipitation in the Black Forest region, as was observed. However, the location and the timing of convection differ substantially between the simulations, and some simulations miss the convective character of the event.

The first row of Fig. 2 shows the simulated precipitation for different setups of the COSMO model. The impact of the starting time of the model simulations and hence, the spin-up prior to the onset of convection, is especially striking (compare the first two figures: IPA-01 was started at 07 UTC, IPA-02 at 00 UTC). The difference between the two COSMO model setups at IPA and IMK can be attributed to a combination of the use of different initial and boundary conditions (GME data were used in the IMK simulations compared to COSMO-EU for the IPA simulations), the choice of different numerical solvers, as well as other details of the model setup. The second row of Fig. 2 depicts three representative members of the COSMO DLR/ MS ensemble. Each of the simulations was started at 06 UTC from different members of the COSMO-LEPS ensemble. Hence, the significant differences between these model simulations are exclusively due to the use of different initial and boundary conditions. The third row of Fig. 2 shows the model results obtained with the MM5 and the WRF models. Comparing the results obtained by the WRF model using initial and boundary data from ECMWF with those obtained using NCEP data, highlights again the importance of the initial and boundary conditions for such convection-resolving model simulations. Other simulations not presented here also indicate that for this case study the choice 

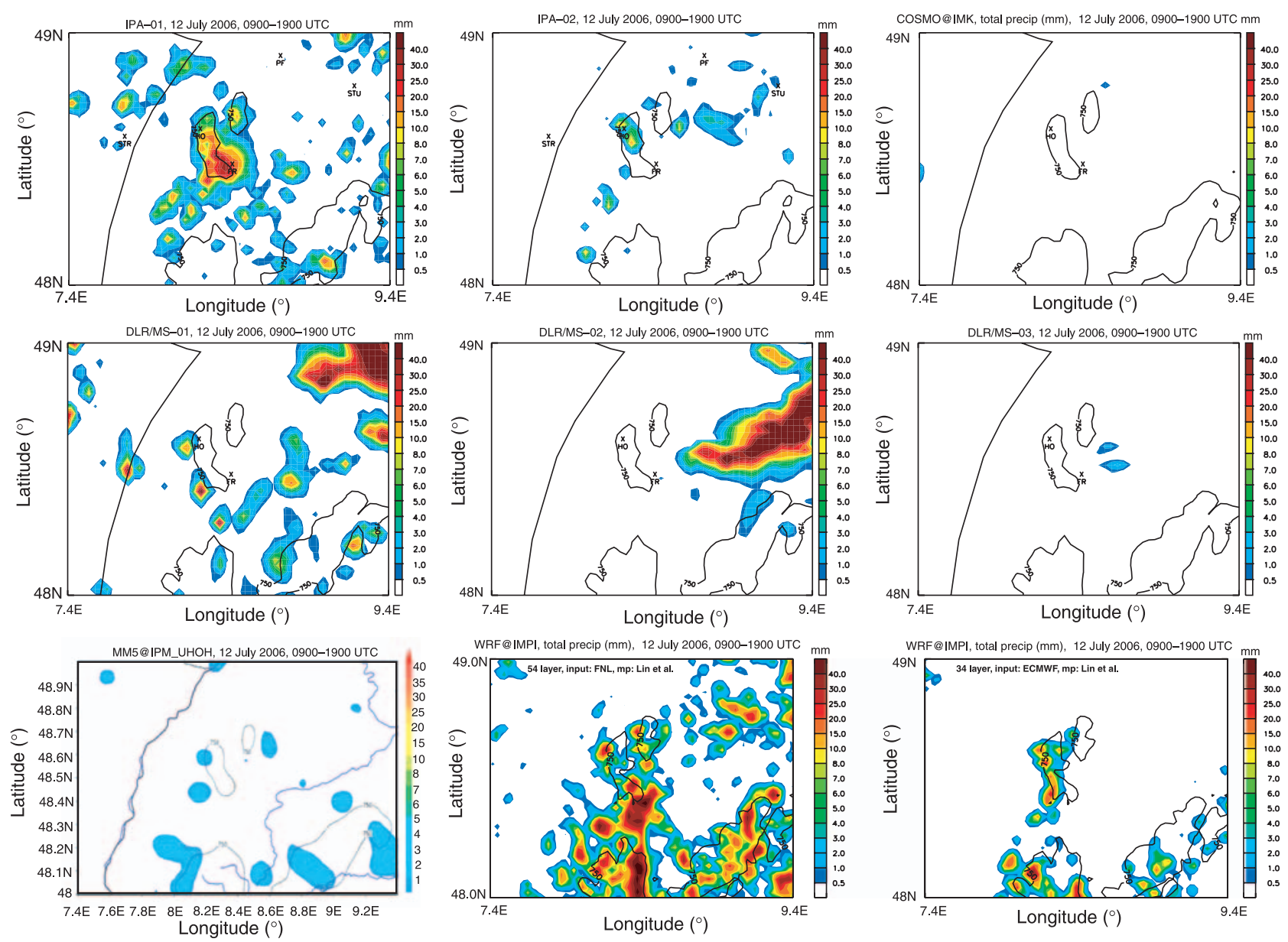

Fig. 2. Simulated precipitation between 09 UTC and 19 UTC for nine model simulations in the Black Forest, black contour line reprents the $750 \mathrm{~m}$-isoline of the topography. First row, left to right: COSMO model, IPA-01, start of the simulation at 07 UTC; COSMO model, IPA-2, start of the simulation at 00 UTC; COSMO model, IMK; second row: three members of the ensemble simulations with the COSMO model, DLR/MS; third row, left to right: MM5, IPM-MM5; WRF, MPI-NCEP; WRF, MPI-ECMWF

of the initial and boundary conditions has a larger impact on the model results than changing parts of the model physics, e.g., the microphysics scheme. The use of ECMWF data in WRF simulations consistently resulted in less precipitation compared to simulations that were driven with NCEP data. However, IPM-MM5 and MPI-ECMWF, which both use initial and boundary conditions based on ECMWF analysis, yield very different precipitation fields, indicating that in this case the impact of using different models (characterized by different grids, numerics, physical parameterizations, etc.) is also substantial.

Most model simulations do not reproduce the details of the observed precipitation pattern, i.e., maximum precipitation inside and close to the
Murg valley, and no precipitation in the Rhine valley. A meaningful, quantitative evaluation of simulated precipitation with observations, e.g., from radar, is not straightforward. Standard evaluation tools, which rely on a point-by-point comparison (e.g., RMSE), are not appropriate for high-resolution model simulations. New tools for the quantitative verification of high-resolution forecasts have recently been developed and tested (Keil and Craig 2007; Wernli et al. 2008). However, it is not the objective of this work to quantitatively verify the performance of the model simulations for this case study. Instead, we focus on the variability of the results obtained from the different model simulations and the applicability of convection indices to analyze the model results. 


\subsection{Diurnal variations and convection indices}

In the following we investigate whether the commonly-used convection indices CAPE and CIN are useful for the diagnosis of convection-resolving model results with respect to the occurrence of convective precipitation. This analysis provides important information about the relevance of these indices for the initiation of convection in high-resolution models.

Figure 3 presents the diurnal variation of the spatially-averaged precipitation (see black box in Fig. 1, right) from the model simulations that

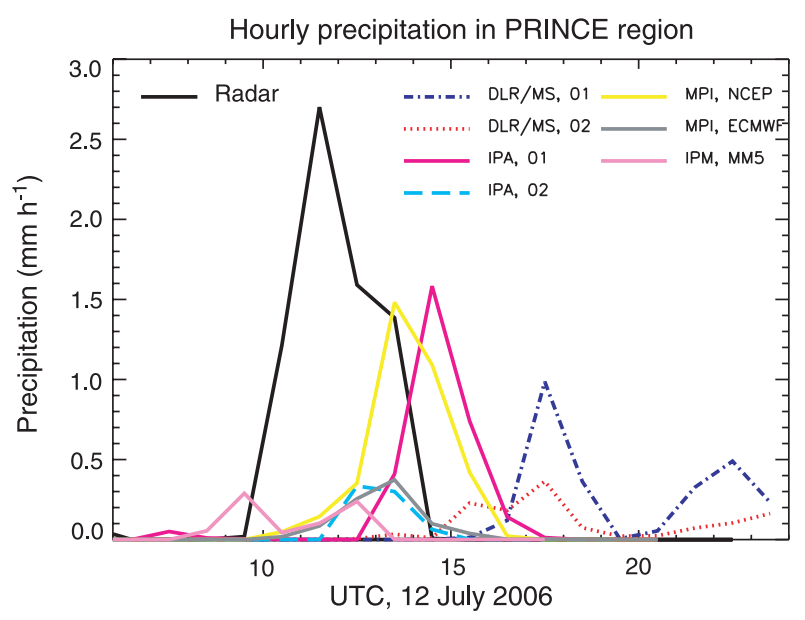

Fig. 3. Spatially-averaged diurnal cycle of precipitation from the model simulations and from radar measurements. The spatial averaging was conducted over the region indicated by the black rectangle in Fig. 1 (right) produce a significant amount of precipitation and from the gauge-adjusted radar observations. The convection observed in this region was initiated by local wind systems in this orographically-structured terrain. The diurnal cycle of precipitation on 12 July 2006 closely follows the typical diurnal cycle of convective activity in the Black Forest region (Finke and Hauf 1996; Aoshima et al. 2008) with a slightly earlier onset.

In most simulations, the onset of precipitation is delayed and all simulations underestimate the amount of precipitation compared to the radar-derived precipitation estimate. While the maximum precipitation of about $2.7 \mathrm{~mm} \mathrm{~h}^{-1}$ was observed between 11 and 12 UTC, most simulations produce the maximum amount of precipitation after 14 UTC. Only the IPM-MM5 model simulation predicts precipitation before 10 UTC; however, the amount is significantly underestimated. In the MPI-NCEP, MPI-ECMWF, and IPA-02 simulations precipitation starts between 12 and 13 UTC, but only the MPI-NCEP simulation results in a significant amount of more than $1 \mathrm{~mm} \mathrm{~h}^{-1}$. The IPA-01 simulation produces the most precipitation in this region (about $1.6 \mathrm{~mm} \mathrm{~h}^{-1}$ ); however, this maximum is delayed by about 3 hours compared to radar observations. The two members of the DLR/MS ensemble produce precipitation after 15 UTC. The DLR/MS01 simulation results in localized cellular precipitation with low spatially-averaged precipitation amounts (see Fig. 2, second row, left). The high
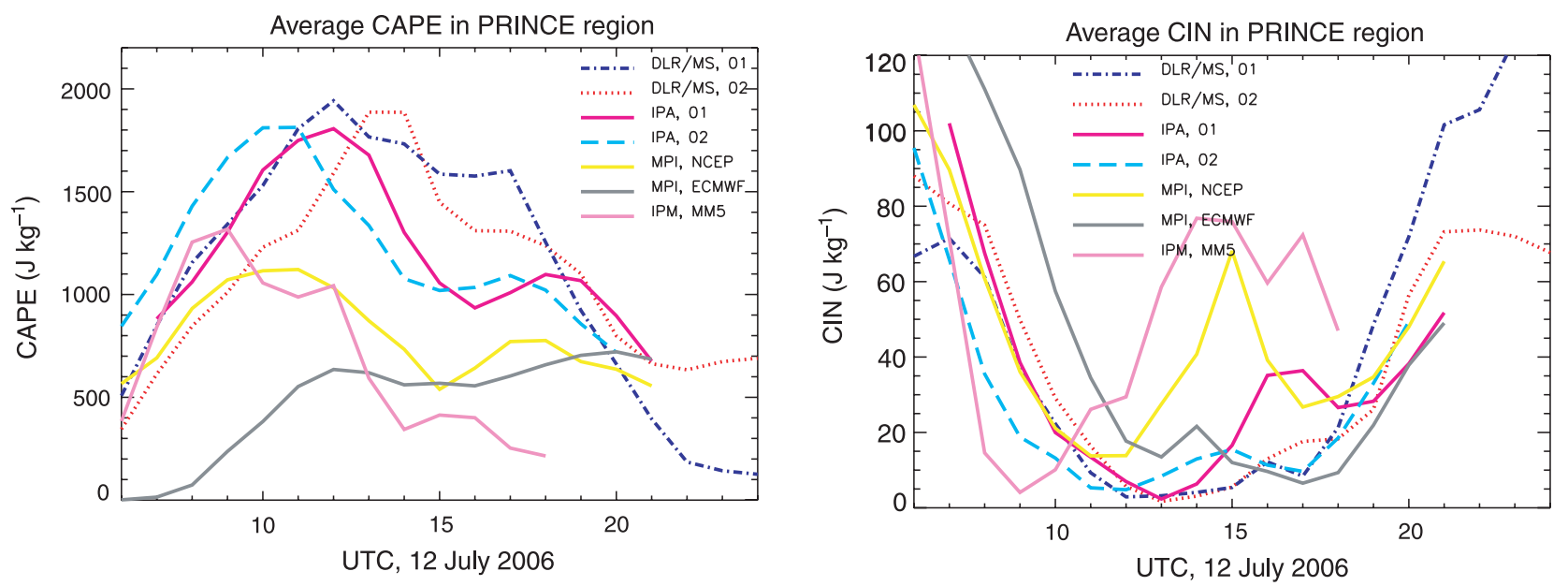

Fig. 4. Spatially-averaged diurnal cycles of (left) CAPE and (right) CIN from the model simulations. The spatial averaging was conducted over the region indicated by the black rectangle in Fig. 1 (right) 
value of the averaged precipitation of the DLR/ MS-02 simulation between 17 and 18 UTC is due to precipitation in the eastern part of the area under consideration, which is not tied to convection initiated by valley-wind circulations in the Murg Valley.

Figure 4 presents the diurnal variations of spatially-averaged values of the convective available potential energy (CAPE) and the convective inhibition (CIN) derived from the model simulations. For the calculation of these indices, the lowest model level was used as initial condition for the parcel ascent. Since precipitation in the Black Forest region was locally initiated, a correlation between convection indices like CAPE or CIN and the onset of surface precipitation can be expected. On a larger scale, this correlation can be degraded due to the propagation of precipitating weather systems into the averaging region. The absolute values of CAPE and CIN from the IPM-MM5 simulation should not be compared quantitatively with the other values, since a different method for the calculation of these indices was used. In the following, we focus on the diurnal cycles of CAPE and CIN and their relation to the diurnal cycle of precipitation.

In general, all model simulations reproduce the expected diurnal cycle of CAPE (CIN) with low (high) values in the morning hours, maximum (minimum) values in the early afternoon, and a decrease (increase) towards the evening. The maximum CAPE values simulated with the COSMO model are very comparable in magnitude with CAPE derived from radiosondes (Sect. 3). The model simulation with the earliest onset of precipitation (IPM-MM5) shows the strongest decrease of CIN and increase of CAPE during the morning hours. The two IPA simulations differ in their timing of the maximum/ minimum value of CAPE/CIN, respectively, and with a corresponding difference in the onset of precipitation (Fig. 3). The CAPE and CIN values of the MPI-ECMWF simulation are significantly different from the corresponding values from the MPI-NCEP simulation, reflecting the relatively large difference between the driving data for these two simulations. However, the timing of the precipitation in these two model simulations is very similar. The diurnal cycles of CAPE and CIN of the DLR/MS-02 simulation are very comparable to those from the IPA-01 simulation, until the onset of precipitation in the IPA-01 simulation between 14 and 15 UTC. The increase (decrease) of CAPE (CIN) in the DLR/MS-01 simulation is delayed compared to the DLR/MS-02 simulation, but comparable in magnitude.

The impact of precipitating deep convection on CAPE and CIN resulting in an increase of CIN and a decrease of CAPE can be seen in all model simulations. This effect is explained by the atmospheric stabilization induced by deep convection, and is most pronounced in the IPMMM5 simulation. There is, however, no clear correlation between the amount of precipitation and the atmospheric stabilization induced by the convection as manifested by the changes of CAPE and CIN. The model simulation with the largest amount of precipitation (IPA-01) shows only modest modifications of CAPE and CIN. The total amount of precipitation is also not well correlated with the absolute values of CAPE and CIN. The model simulations from DLR/MS and IPA show very comparable values of CAPE and CIN. While the onset of precipitation in these model simulations is consistent with the diurnal cycle of CAPE and CIN, the amount of precipitation differs substantially.

We conclude that the convection indices like CAPE and CIN are useful qualitative proxies for diagnosing the occurrence of convective precipitation in convection-resolving models. However, there is no unambiguous relationship between these indices and the onset of convective precipitation, suggesting that other atmospheric factors (e.g., the atmospheric moisture content, low level wind convergence) are also relevant. The amount of precipitation does not seem to be correlated with these convection indices in the model simulations.

\section{Conclusions}

A first comparison has been presented of the performance of three convection-resolving models (the COSMO, MM5, and the WRF model) for a convective scenario with weak synoptic-scale forcing. On 12 July 2006, local convective cells formed in mountainous regions across central Europe, including the Black Forest. The model comparison was limited to a relatively small region in the northern part of the Black Forest and 
aimed at the evaluation of the diurnal cycles of convective precipitation and the convective indices CAPE and CIN.

The models were driven by different meteorological data and were run with different setups by several research groups. The simulated convective precipitation on 12 July 2006 was found to be very sensitive to the model configuration, the initial and boundary conditions, and the spin-up time before the convection. Six out of nine simulations capture the convective character of the event. However, they differ in the location and timing of the intense convective cells. Three simulations fail to capture the convective nature of this event.

Analysis of the diurnal cycle of precipitation showed that only one simulation realistically represents the early onset time of precipitation. All simulations underestimate the amount of convective precipitation. The simulated diurnal cycles of CAPE (CIN) showed the expected variations with maximum (minimum) values in the early afternoon. For a given model setup, the diurnal cycles of CAPE and CIN are clearly related to the onset of precipitation. The significance of this relation, also for other cases in different regions, requires further investigations. However, there is no correlation between the values of CAPE and/or CIN with the amount of precipitation.

The results obtained for this case study show that the deterministic simulation, and hence forecasting, of local convection in low-mountain terrain using convection-resolving models is extremely challenging. Current state-of-the-art models are capable of reproducing the convective nature of the event studied here, but details like the onset (time and location) and the amount of convective precipitation are not well simulated. For this case study, the initial and lateral boundary conditions used for the high-resolution model simulations had a large impact on the simulated precipitation.

The development and application of advanced data assimilation techniques for convection-resolving models might improve the simulations. For the event on 12 July 2006, radar rainfall data assimilation by latent heat nudging forced all ten members of the high resolution ensemble system employed at DLR/MeteoSwiss to generate convective precipitation as shown in an independent study (Keil et al. 2007). Highresolution ensemble prediction systems, which include perturbations of the initial and boundary conditions and of the model physics can also provide valuable guidance for improved weather forecasting.

\section{Acknowledgements}

We thank Elmar Weigl, DWD, and MeteoSwiss for kindly providing the radar data and the MSG satellite image, respectively. J.T., C.B. and C.K. thank the DWD for providing and supporting the use of the COSMO model. This work was funded by the Helmholtz Association (Virtual Institute COSI-TRACKS). We thank two anonymous reviewers for their constructive comments that helped to improve the manuscript.

\section{References}

Aoshima F, Behrendt A, Bauer H-S, Wulfmeyer V (2008) Statistics of convection initiation by use of Meteosat Rapid Scanning Data during the Convective and Orographically-Induced Precipitation Study (COPS). Meteorol Z (submitted)

Didone M, Lüthi D, Davies HC (2007) Some factors in the design of a regional prediction model: an examination based upon two MAP events. Meteorol Z 16: 261-73

Finke U, Hauf T (1996) The characteristics of lightning occurence in Southern Germany. Beitr Phys Atmos 69: 361-74

Grell G, Dudhia J, Stauffer D (1995) A description of the fifth-generation Penn State/NCAR Mesoscale Model (MM5). NCAR Tech Notes 398+ STR, NCAR, Boulder, CO

Groenemeijer P, Barthlott Ch, Behrendt A, Corsmeier U, Handwerker J, Kohler M, Kottmeier Ch, Mahlke H, Pal S, Radlach M, Trentmann J, Wieser A, Wulfmeyer V (2008) Multisensor measurements of a convective storm cluster over a low mountain range: adaptive observations during PRINCE. Mon Wea Rev (submitted)

Grzeschik M, Bauer H-S, Wulfmeyer V, Engelbart D, Wandinger U, Mattis I, Althausen D, Engelmann R, Tesche M, Riede A (2008) Four-dimensional variational analysis of water-vapor Raman lidar data and their impact on mesoscale forecasts. J Atmos Oceanic Technol (in press)

Hohenegger C, Lüthi D, Schär C (2006) Predictability mysteries in cloud-resolving models. Mon Wea Rev 134: 2095-107

Keil C, Craig GC (2007) A displacement-based error measure applied in a regional ensemble forecasting system. Mon Wea Rev 135: 3248-59

Keil C, Leuenberger D, Craig GC (2007) Radar rainfall assimilation and short-range QPF in a high-resolution EPS, Extended abstract, ICAM 2007

Leuenberger D, Rossa A (2007) Revisiting the latent heat nudging scheme for the rainfall assimilation of 
a simulated convective storm. Meteorol Atmos Phys 98: 195-215

Molteni F, Buizza R, Marsigli C, Montani A, Nerozzi F, Paccagnella T (2001) A strategy for high-resolution ensemble prediction. Part I: Definition of representative members and global-model experiments. Quart J Roy Meteor Soc 127: 2069-94

Schättler U, Doms G, Schraff C (2005) A description of the nonhydrostatic regional model LM, Part VII: User's guide. Consortium for Small-Scale Modelling

Schwitalla T, Bauer H-S, Wulfmeyer V, Zängl G (2008) Systematic errors of QPF in low-mountain regions. Meteorol Z (submitted)

Skamarock WC, Klemp JB, Dudhia J, Gill DO, Barker DM, Wang W, Powers JG (2005) A description of the advanced research WRF Version V2, NCAR/TN486/STR, Boulder, CO, USA

Steppeler J, Doms G, Schättler U, Bitzer HW, Gassmann A, Damrath U, Gregoric G (2003) Meso-gamma scale forecasts using the nonhydrostatic model LM. Meteorol Atmos Phys 82: 75-96

Wernli H, Paulat M, Hagen M, Frei C (2008) SAL - a novel quality measure for the verification of quantitative precipitation forecasts. Mon Wea Rev (in press)

Wulfmeyer V, Bauer H-S, Grzeschik M, Behrendt A, Vandenberghe F, Browell EV, Ismail S, Ferrare RA (2006) Four-dimensional variational assimilation of water vapor differential absorption lidar data: the first case study within IHOP_2002. Mon Wea Rev 134: 209-30 